\title{
Two-Year Follow-Up After Endovascular Therapy of Superficial Femoral Arteries with Retrograde Popliteal Approach: Single-Center Experience
}

\author{
Emced Khalil, ${ }^{1}$ Sedat Ozcan ${ }^{2}$ \\ ${ }^{1}$ Department of Cardiovascular Surgery, Ordu University Research and Education Hospital, Ordu, Turkey; ${ }^{2}$ Department of \\ Cardiovascular Surgery, Çanakkale 18 Mart University Faculty of Medicine, Çanakkale, Turkey
}

\section{ABSTRACT}

Objective: Popliteal artery puncture, which makes possible the use of lower profile sheaths and devices, has gained popularity as an alternative to the antegrade approach, due to the reduction in vascular complications at the access site. The present study aimed to analyze the safety of the procedure and long-term patency of the superficial femoral artery (SFA) and popliteal artery (PA) and in subjects undergoing recanalization with the popliteal retrograde approach.

Methods: Forty-three subjects, who underwent endovascular therapy (EVT) of the SFA or PA with retrograde popliteal approach, were enrolled in this retrospective study. The decision for the retrograde approach was made according to pre-intervention CT angiography results (severe calcification and relatively long CTO segment) in 20 of the subjects. The remaining 23 subjects underwent SFA or PA recanalization with the retrograde approach, due to failed antegrade recanalization attempt. All patients underwent color duplex ultrasound at the first, sixth, 12th, and 24th months to determine patency. The rate of procedural complications, including hematoma, bleeding, and distal embolism, were recorded for all subjects.

Results: Technical success was achieved in all cases. Access site complications, including hematoma and bleeding, were observed in 2 subjects (4.66\%). Transfusion or surgical treatments were not required in any cases with access site complications. The acute success rate was defined as the recovery of good blood flow evaluated by angiography after EVT was $100 \%$. Distal embolization, which did not cause any limitations in distal flow, occurred in 1 subject (2.33\%). Anklebrachial index (ABI) calculated at 1 month post-intervention was significantly higher than pre-intervention ABI [0.9 (0.59 - 1.3$)$ versus 0.7 (0.4 - 1.1), $P<.001]$. Patency rates, as determined by ultrasonographic assessment at post-interventional first, sixth, 12th, and 24th months, were 100\%, 95.34\%, $88.37 \%$, and $86.04 \%$, respectively.

Conclusion: Our findings demonstrate that retrograde popliteal artery puncture can be used as a safe and effective

Received April 9, 2020; received in revised form April 15, 2020; accepted April $15,2020$.

Correspondence: Emced Khalil, Department of Cardiovascular Surgery, Ordu University Research and Education Hospital, Ordu, Turkey. (e-mail: emjedkhalil@gmail.com). technique for recanalization of SFA and PA stenosis and occlusions. The retrograde popliteal approach provides excellent long-term primary patency rates.

\section{INTRODUCTION}

Femoropopliteal atherosclerotic disease constitutes the majority of lower extremity peripheral artery diseases. The superficial femoral artery (SFA) is located within a fibromuscular canal, which exposes this artery to highly dynamic forces of flexion, extension, shortening, and torsion [Yahagi 2014]. This unique location of the SFA and associated mechanical forces are responsible for chronic vascular injuries stemming from cyclic deformation, strain, and cellular proliferation [Banerjee 2016]. Moreover, disorders in the expression of several proatherogenic genes are hypothesized to contribute to the development of SFA stenosis. These mechanical forces and resultant repetitive vascular injury not only makes SFA prone to de novo atherosclerosis, but also facilitates restenosis in subjects undergoing SFA revascularization [Ansari 2013].

The recommended first-line revascularization strategy in TASC II class D SFA occlusions is femoropopliteal bypass surgery [Norgren 2007]. This recommendation primarily is based on the variable (often lower) long-term patency rates observed with endovascular therapy (EVT) compared with surgical revascularization. Extensive lesion length, presence of micro- and macro-dissections, vessel size, and limited capability of the artery for positive remodeling all contribute to poor patency rates after EVT. However, recent advances in endovascular technology enable high success rates (exceeding $80 \%-90 \%$ ) with EVT in TASC II C/D lesions [Goltz 2015]. Contralateral retrograde puncture or ipsilateral antegrade puncture of the common femoral artery frequently are employed, when performing antegrade approach EVT. However, antegrade puncture is not feasible in case of a blunted, calcified proximal cap, which makes guidewire passage complicated, and in subjects with a proximal SFA disease, in whom sheath replacement is problematic due to the short distance between the access point and the proximal cap of the SFA lesion. Popliteal artery puncture, which makes possible the use of lower profile sheaths and devices, recently has gained popularity as an alternative to the antegrade approach, particularly due to reportedly reduced risks for vascular complications at the access site [Ueshima 2015]. 
Although there are several reports indicating that the retrograde approach is a safe and reliable option in recanalization of the SFA, there still are some controversies, regarding long-term outcomes. The present study aimed to analyze the safety and long-term patency of the SFA in subjects undergoing recanalization with the popliteal retrograde approach.

MATERIALS AND METHODS

\section{Subjects}

Forty-three consecutive patients who underwent retrograde recanalization of the SFA or PA for partial stenosis or chronic total occlusion at Ordu Training and Research Hospital between August 2015 and August 2017 were enrolled in this retrospective study. SFA or PFA disease was confirmed by MR angiography or CT angiography in subjects with risk factors for atherosclerotic vascular disease and symptoms of stenotic peripheral arterial disease, including rest pain, claudication, and cold lower extremities. Additionally, all subjects underwent digital subtraction angiography for a detailed description of lesion characteristics and identification of the collateral blood supply just before the intervention. Written informed consent was obtained from all participants. The study was approved by the Institutional Review Board and was conducted by the Helsinki declaration. Subjects with a history of prior SFA or PA intervention or surgery, previous coronary artery bypass grafting surgery, and those with contraindications to antiaggregant agents were excluded from the study. All required data were retrieved from the institutional digital database and patient charts.

\section{Intervention}

The same interventional team performed all EVTs. The retrograde approach was preferred in 20 subjects, according to pre-interventional CT angiography results (severe calcification and relatively long CTO segment). The remaining 23 subjects underwent SFA recanalization with a retrograde approach, due to the failure of antegrade recanalization. The popliteal artery was selected as the puncture site in all subjects. Popliteal artery puncture was carried out with a 20 or 22 $G$ needle under ultrasonographic guidance with the patient in the prone position. A $6 \mathrm{~F}-7 \mathrm{~F}$ sheath or a microcatheter was placed into the popliteal artery, and $100 \mathrm{U} / \mathrm{kg}$ intravenous UF heparin was administered to achieve adequate anticoagulation during the intervention. A 0.014 or 0.018 guidewire was advanced through the occlusion, and recanalization of the SFA and PA stenosis were performed using paclitaxel-coated balloons. Upon the completion of the procedure, the sheath was replaced with a 5F-sized sheath. Vascular sheaths were left in the artery for 4 hours after the procedure and were then removed. Hemostasis was achieved by manual compression, during sheath removal. For procedures in which a sheath was not used, we also applied compression on the popliteal artery for hemostasis-a combination-treated patients of 2 antiplatelet drugs: cilostazol and clopidogrel after the procedure. Additionally, low-molecular-weight heparin was
Table 1. Summary of Patient Characteristics

\begin{tabular}{lc}
\hline Age (years, mean \pm SD) & $64.14 \pm 9.18$ \\
Gender (male) & $39(90.70 \%)$ \\
Rutherford Classification & \\
3 & $20(46.51 \%)$ \\
4 & $21(48.84 \%)$ \\
5 & $2(4.65 \%)$ \\
TASC II & \\
B & $25(58.14 \%)$ \\
C & $12(27.91 \%)$ \\
D & $6(13.95 \%)$ \\
Smokers & $18(41.86 \%)$ \\
Hyperlipidemia & $13(30.23 \%)$ \\
Diabetes Mellitus & $20(46.51 \%)$ \\
Hypertension & $36(83.72 \%)$ \\
Chronic Renal Failure & $2(4.65 \%)$ \\
COPD & $4(9.30 \%)$ \\
Coronary Artery Disease & $12(27.91 \%)$ \\
Indication & \\
Claudication & $16(37.21 \%)$ \\
Rest Pain & $26(60.47 \%)$ \\
Wound & $1(2.33 \%)$ \\
\hline
\end{tabular}

Data are given as mean \pm standard deviation or median (minimum - maximum) for continuous variables with regard to normality and frequency COPD: Chronic Obstructive Pulmonary Disease

prescribed for 2 weeks. All patients received the maximum tolerated dose of statins.

\section{Primary Outcome}

All subjects underwent physical examination and color duplex ultrasound at first, sixth, 12th, and 24th months to evaluate the patency of the relevant SFA. The rate of primary patency, which was defined as the complete lack of restenosis at the target lesion, was the primary outcome measure, during the follow-up evaluations. The rate of procedural complications, including hematoma, bleeding, and distal embolism, were secondary outcome measures.

\section{Statistical Analysis}

All analyses were performed on SPSS v21 (SPSS Inc., Chicago, IL). For the normality check, the Shapiro-Wilk test was used. Data are given as mean \pm standard deviation or median (minimum-maximum) for continuous variables concerning the normality of distribution and as frequency (percentage) for categorical variables. Non-normally distributed variables (ankle-brachial index) were analyzed with the Wilcoxon Signed Ranks test for paired comparisons. Repeated measures of categorical variables were analyzed with the 
Table 2. Angiographic characteristics

\begin{tabular}{|c|c|}
\hline Length of Stenosis $(\mathrm{cm})$ & $10(4-20)$ \\
\hline Directional Atherectomy & $17(39.53 \%)$ \\
\hline \multicolumn{2}{|l|}{ Lesion Characteristic } \\
\hline \multicolumn{2}{|l|}{ (Occlusion) } \\
\hline Partial (>70\% stenosis) & $10(23.26 \%)$ \\
\hline Total occlusion & $33(76.74 \%)$ \\
\hline \multicolumn{2}{|l|}{ Localization } \\
\hline SFA & $25(58.10 \%)$ \\
\hline SFA + POPL & $16(39.02 \%)$ \\
\hline SFA + Iliac & $2(4.88 \%)$ \\
\hline \multicolumn{2}{|l|}{ Length of Balloon (mm) } \\
\hline 40 & $1(2.33 \%)$ \\
\hline 60 & $3(6.98 \%)$ \\
\hline 80 & $6(13.95 \%)$ \\
\hline 100 & $15(34.88 \%)$ \\
\hline 150 & $18(41.86 \%)$ \\
\hline \multicolumn{2}{|l|}{ Diameter of Balloon (mm) } \\
\hline 4 & $12(27.91 \%)$ \\
\hline 5 & $20(46.51 \%)$ \\
\hline 6 & $11(25.58 \%)$ \\
\hline Retrograde Approach & $43(100.00 \%)$ \\
\hline \multicolumn{2}{|l|}{ Sheath } \\
\hline $6 \mathrm{~F}$ & $25(58.14 \%)$ \\
\hline $7 F$ & $18(41.86 \%)$ \\
\hline Microcatheter alone & $12(27.91 \%)$ \\
\hline
\end{tabular}

Cochran's Q test. A $P$-value of $<.05$ was accepted to demonstrate statistical significance.

\section{RESULTS}

Longitudinal data was available for 43 subjects (mean age $64.14 \pm 9.18$ years, $90.7 \%$ male). Twelve of the subjects $(27.91 \%)$ had preexisting coronary artery disease. Pure SFA lesions were noted in 25 (58.10\%) of the subjects, whereas SFA lesions were extending to the popliteal artery in $16(39.02 \%)$ patients. One percent of the SFA lesions were Rutherford category 3, $48.8 \%$ were Rutherford category 4, and $4.6 \%$ were Rutherford category 5. TASC II classifications of the SFA lesions were as follows: $58.14 \%$ class B, $27.91 \%$ class C, and $13.95 \%$ class D. The median length of SFA lesions was 10 $(4-20) \mathrm{cm}$. The lengths of the balloons used for intervention are presented in Table 1. Retrograde popliteal puncture was successful in all subjects. Directional atherectomy was applied in $39.53 \%$ of the interventions. SFA lesions were symptomatic in all subjects, with resting pain being the most common symptom (60.47\%). Access site complications, including hematoma and bleeding, were observed in 2 subjects $(4.66 \%)$.
Table 3. Primary and secondary outcomes

$\begin{array}{lc}\text { Complications } & \\ \text { None, } \mathrm{n} & 40(93.02 \%) \\ \text { Hematoma, } \mathrm{n} & 1(2.33 \%) \\ \text { Bleeding, } \mathrm{n} & 1(2.33 \%) \\ \text { Embolism, } \mathrm{n} & 1(2.33 \%) \\ \text { Ankle-Brachial Index } & \\ \text { Pre-intervention } & 0.7(0.4-1.1) \\ \text { Post-intervention } & 0.9(0.59-1.3) \\ & P<.001 \\ \text { Primary patency rate } & \\ \text { 1st Month, } \mathrm{n} & 43(100.00 \%) \\ \text { 6th Month, } \mathrm{n} & 41(95.34 \%) \\ \text { 12th Month, } \mathrm{n} & 38(88.37 \%) \\ \text { 24th Month, } \mathrm{n} & 37(86.04 \%)\end{array}$

Transfusion or surgical treatments were not required in any of the cases with access site complications. Acute success rate, which was defined as the recovery of good blood flow evaluated by angiography after EVT, was $100 \%$. Distal embolization, which did not cause any limitations in distal flow, occurred in 1 subject $(2.33 \%)$. There were no peri-procedural complications in $40(93.02 \%)$ of the subjects. Ankle-brachial index $(\mathrm{ABI})$ values, calculated 1 month after the intervention, were significantly higher than pre-intervention $\mathrm{ABI}$ values [0.9 (0.59 - 1.3) versus $0.7(0.4-1.1), P<.001]$. The patency rates indicated by the ultrasonographic assessment at first, sixth, 12th, and 24th months after intervention were $100.00 \%, 95.34 \%, 88.37 \%$, and $86.04 \%$, respectively. A small percentage of subjects underwent re-intervention due to the restenosis at the 1st (2 subjects) and 6th months (3 subjects). One subject was referred to surgery for the restenosis at 12 th month for which interventional attempt to recanalize the restenotic lesion was unsuccessful. None of the re-occlusions resulted in mortality or amputation.

\section{DISCUSSION}

The present single-center study demonstrates that EVT with a retrograde popliteal approach provides not only acute success in restoration of distal flow, but also ensures excellent long-term patency rates. Access site complication rate also is acceptable, and these complications are defined as minor complications that do not necessitate transfusion or surgical treatments. Additionally, it is apparent that EVT with a retrograde popliteal approach leads to a significant increase in ABI. These findings confirm that EVT with retrograde popliteal puncture is an effective and safe approach in the management of complex SFA occlusions.

ACC/AHA Practice Guidelines for the management of patients with peripheral arterial disease indicate that procedural success rates and 12 months patency rates after femoral 
artery interventions are $75 \%-90 \%$ and $26 \%-80 \%$, respectively [Hirsch 2006]. High calcium content, long length of lesions, and different dynamic forces found within these arteries are the major contributors to the procedural success rate and longterm patency rate of SFA interventions [Dumantepe 2017].

Consequently, surgery still is recommended as the preferred treatment modality in TASC II C and D lesions, which present with excessive calcification. However, with the recent advances in the guidewire, microcatheter, and balloon technology, EVT is emerging as a possible primary option for the management of infrainguinal peripheral occlusive disease. Recent evidence indicates that EVT appears feasible in the management of $>90 \%$ of femoropopliteal occlusions [Conrad 2006]. The majority of SFA lesions are treated by an antegrade ipsilateral or retrograde contralateral femoral approach. Retrograde popliteal approach, which was initially considered as a 'backup' option, is now being increasingly employed as a first choice vascular access option, particularly in proximal lesions of the SFA with no stump, tandem iliac, and SFA lesions, and particularly in patients with severe obesity [Brountzos 2011; Schmidt 2012; Kawarada 2011]. Also, the antegrade approach may be challenging in cases with narrow aortic bifurcation and in subjects with a history of abdominal aortic aneurysm repair. Although the ipsilateral antegrade approach offers better instrumentation and is primarily chosen for this feature, it cannot be performed in the presence of lesions involving the CFA.

The retrograde popliteal approach was first described by Tonnesen et al in 1988. The popularity of this approach increased, following the description of safe techniques for popliteal artery puncture and several guidance methods facilitating popliteal artery puncture, including ultrasound and fluoroscopic guidance [Trigaux 1991]. Previous data reports that retrograde popliteal approach might be useful in the failure of antegrade recanalization, typical femoral artery occlusion, SFA occlusions with a small stump, in cases where entering the true lumen distal to the obstruction is unfeasible, and in cases with tandem lesions [Saha 2001; Heenan 1994; Henry 1993].

In the present study, 23 of the subjects had a history of failed antegrade recanalization attempt, and $48 \%$ were TASC II C and D lesions. The median length of the SFA lesions was $10(4-20) \mathrm{cm}$. Despite the challenging lesion characteristics, the technical success rate, including puncture of the popliteal artery and successful recanalization of the SFA, was $100 \%$. In a recent study conducted by Ueshima and colleagues, the primary success rate of SFA occlusion with the retrograde popliteal approach was 97.2\% [Ueshima 2015]. Another study conducted by Ye et al demonstrated a technical success rate of $100 \%$ in subjects undergoing EVT with a retrograde popliteal approach, due to a failed antegrade SFA recanalization attempt [Ye 2013].

A similar technical success rate also was reported by the study of Dumantepe at el in which retrograde popliteal artery access was employed for the treatment of SFA occlusions. In that study, the authors reported patency rates of $100 \%$, $92.8 \%$, and $85.7 \%$ with follow-up evaluations performed at 1 , 6 , and 12 months after intervention [Dumantepe 2017]. The primary patency rate with the retrograde popliteal approach reported by Ye et al at 6 months was $84.2 \%$. WojtasikBakalarz et al, in their recent study, reported that the primary patency rate at 12 months was $88.2 \%$ in subjects undergoing EVT with retrograde popliteal approach after failed percutaneous antegrade recanalization [Wojtasik-Bakalarz 2017]. In our study, the primary patency rates of the subjects undergoing SFA intervention with the retrograde popliteal approach at 6,12 , and 24 months were $95.34 \%, 88.37 \%$, and $86.04 \%$, respectively. Compared with previous data, the primary patency rates at the follow-up period of our study were somewhat higher. One possible explanation for this might be the enrollment of 10 subjects with partial SFA stenosis. Routine use of paclitaxel-coated balloons also might have a role in favorable primary patency rates.

Popliteal puncture also seems safe, in terms of access site complications. In our study, 1 (2.3\%) bleeding event and 1 $(2.3 \%)$ hematoma event occurred, following the removal of the sheath. Both complications were managed with prolonged manual compression. None of the subjects required transfusion or surgery for the management of these minor access site complications. Our findings regarding the complication rates confirm the safety of the retrograde popliteal approach reported in previous studies, which have found complication rates at a range of $3.3-27.7 \%$, depending on the definition used to identify complications [Saha 2001; Henry 1993; Zaitoun 1990; Yilmaz 2005].

Routine ultrasound-guided access previously has been shown to reduce the rate of puncture-related complications [Yilmaz 2005]. We believe that the placement of a smaller size sheath before sheath removal and ultrasonographic guidance during vascular entry may contribute to reduced puncturerelated complication rates in subjects undergoing SFA intervention with the retrograde popliteal approach.

We also observed that preprocedural CT angiographic evaluation of the lesion characteristics led to a shorter intervention time and, accordingly, less X-ray exposure. We suggest that CT angiography should be performed in all subjects scheduled for peripheral artery intervention to reduce the $\mathrm{X}$-ray exposure.

The present study has some limitations to be mentioned. The retrospective design, relatively small sample size, and single-center data are the major drawbacks of our research. The lack of the assessment of clinical signs and symptoms related to SFA stenosis also is a limitation for this study. However, by design, this study aimed to determine the success with a retrograde approach. Nevertheless, clinical data may have contributed to the understanding of the relationship between success rates and disease severity, even though all patients had significant complaints. Third, follow-up patency assessment was performed with ultrasonographic evidence only. Given the limitations of ultrasonographic patency assessment (also though it is often very reliable), the adoption of CT angiography could have provided additional information regarding vascular patency.

\section{CONCLUSION}

Our findings demonstrate that the retrograde popliteal artery approach can be used as a safe and effective technique 
for recanalization of SFA and PA stenosis and occlusion. The retrograde popliteal approach also provides excellent longterm primary patency rates. We suggest that this approach be taken into account as a primary option, not only in cases with failed antegrade plan, but also in cases with high risk for access site complications.

\section{REFERENCES}

Ansari F, Pack LK, Brooks SS, Morrison TM. 2013. Design considerations for studies of the biomechanical environment of the femoropopliteal arteries. J Vasc Surg 58(3): 804-13.

Banerjee S. 2016. Superficial Femoral Artery Is Not Left Anterior Descending Artery. Circulation 134(13): 901-3.

Brountzos EN, Moulakakis KG, Avgerinos ED, Dalainas I, T GG, Kakisis J, et al. 2011. Retrograde transpopliteal approach of iliofemoral lesions. Vascular and endovascular surgery 45(7): 646-50.

Conrad MF, Cambria RP, Stone DH, Brewster DC, Kwolek CJ, Watkins MT, et al. 2006. Intermediate results of percutaneous endovascular therapy of femoropopliteal occlusive disease: a contemporary series. J Vasc Surg 44(4): 762-9.

Dumantepe M. 2017. Retrograde Popliteal Access to Percutaneous Peripheral Intervention for Chronic Total Occlusion of Superficial Femoral Arteries. Vascular and endovascular surgery 51(5): 240-46.

Goltz JP, Kleemann M. 2015. Complex recanalization techniques for complex femoro-popliteal lesions: how to optimize outcomes. J Cardiovasc Surg (Torino) 56(1): 31-41.

Heenan SD, Vinnicombe SJ, Buckenham TM, Belli AM. 1994. Percutaneous transluminal angioplasty by a retrograde subintimal transpopliteal approach. Clin Radiol 49(11): 824-7; discussion 27-8.

Henry M, Amicabile C, Amor M, Beron R, Henry I, Mentre B. 1993. [Peripheral arterial angioplasty: value of the popliteal approach. Apropos of 30 cases]. Archives des maladies du coeur et des vaisseaux 86(4): 463-9. 1993/04/01.

Hirsch AT, Haskal ZJ, Hertzer NR, Bakal CW, Creager MA, Halperin JL, et al. 2006. ACC/AHA 2005 Practice Guidelines for the management of patients with peripheral arterial disease (lower extremity, renal, mesenteric, and abdominal aortic): a collaborative report from the American Association for Vascular Surgery/Society for Vascular Surgery, Society for Cardiovascular Angiography and Interventions, Society for Vascular Medicine and Biology, Society of Interventional Radiology, and the ACC/AHA Task Force on Practice Guidelines (Writing Committee to
Develop Guidelines for the Management of Patients With Peripheral Arterial Disease): endorsed by the American Association of Cardiovascular and Pulmonary Rehabilitation; National Heart, Lung, and Blood Institute; Society for Vascular Nursing; TransAtlantic Inter-Society Consensus; and Vascular Disease Foundation. Circulation 113(11): e463-654.

Kawarada O. 2011. Commentary: Miniaturized retrograde popliteal approach in a supine patient. Journal of endovascular therapy : an official journal of the International Society of Endovascular Specialists 18(4): 510-2.

Norgren L, Hiatt WR, Dormandy JA, Nehler MR, Harris KA, Fowkes FG. 2007. Inter-Society Consensus for the Management of Peripheral Arterial Disease (TASC II). J Vasc Surg 45 Suppl S: S5-67.

Saha S, Gibson M, Magee TR, Galland RB, Torrie EP. 2001. Early results of retrograde transpopliteal angioplasty of iliofemoral lesions. Cardiovasc Intervent Radiol 24(6): 378-82.

Schmidt A, Bausback Y, Piorkowski M, Werner M, Braunlich S, Ulrich $\mathrm{M}$, et al. 2012. Retrograde recanalization technique for use after failed antegrade angioplasty in chronic femoral artery occlusions. Journal of endovascular therapy : an official journal of the International Society of Endovascular Specialists 19(1): 23-9.

Trigaux JP, Van Beers B, De Wispelaere JF. 1991. Anatomic relationship between the popliteal artery and vein: a guide to accurate angiographic puncture. AJR Am J Roentgenol 157(6): 1259-62.

Ueshima D, Ashikaga T, Shimura T, Hatano Y, Sasaoka T, Kurihara K, et al. 2015. Popliteal Retrograde Approach is Effective and Safe for Superficial Femoral Artery Chronic Total Occlusion. Ann Vasc Dis 8(3): 220-6.

Wojtasik-Bakalarz J, Arif S, Chyrchel M, Rakowski T, Bartus K, Dudek D, et al. 2017. Twelve months follow-up after retrograde recanalization of superficial femoral artery chronic total occlusion. Postepy Kardiol Interwencyjnej 13(1): 47-52.

Yahagi K, Otsuka F, Sakakura K, Sanchez OD, Kutys R, Ladich E, et al. 2014. Pathophysiology of superficial femoral artery in-stent restenosis. J Cardiovasc Surg (Torino) 55(3): 307-23.

Ye M, Zhang H, Huang X, Shi Y, Yao Q, Zhang L, et al. 2013. Retrograde popliteal approach for challenging occlusions of the femoral-popliteal arteries. J Vasc Surg 58(1): 84-9.

Yilmaz S, Sindel T, Luleci E. 2005. Ultrasound-guided retrograde popliteal artery catheterization: experience in 174 consecutive patients. Journal of endovascular therapy : an official journal of the International Society of Endovascular Specialists 12(6): 714-22.

Zaitoun R, Iyer SS, Lewin RF, Dorros G. 1990. Percutaneous popliteal approach for angioplasty of superficial femoral artery occlusions. Catheterization and cardiovascular diagnosis 21(3): 154-8. 